\title{
nec1, a Gene Conferring a Necrogenic Phenotype, Is Conserved in Plant-Pathogenic Streptomyces spp. and Linked to a Transposase Pseudogene
}

\author{
Raghida A. Bukhalid, Soo Young Chung, and Rosemary Loria \\ Department of Plant Pathology, Cornell University, Ithaca, NY 14853-4203, U.S.A. \\ Accepted 29 June 1998.
}

We are investigating the genetic basis for, and evolution of, plant pathogenicity in Streptomyces spp. The plantpathogenic species $S$. scabies, $S$. acidiscabies, and $S$. turgidiscabies cause the scab disease of potato and produce the phytotoxins, thaxtomins. Forty-three Streptomyces strains representing the three species were evaluated; all thaxtomin A-producing Streptomyces strains were pathogenic on potato tubers and all but one hybridized to nec1 and ORFtnp, two genes previously cloned from S. scabies ATCC 41973. necl confers a pathogenic phenotype on $S$. lividans TK24, a nonpathogen, and ORFtnp is a transposase pseudogene located $5^{\prime}$ to necl. The eight nonpathogenic strains tested neither produced thaxtomin A nor hybridized to nec1. ORFtnp and necl occurred on a single $P v u$ II restriction fragment in all thaxtomin A-producing Streptomyces strains. The nucleotide sequences of the homologs of nec1 and ORFtnp from two pathogenic strains each of S. scabies, S. acidiscabies, and S. turgidiscabies were identical; oligonucleotide primers specific to this gene amplified homologs from all strains that hybridized to nec1. We propose that nec1 and ORFtnp have been horizontally mobilized from $S$. scabies to $S$. acidiscabies and $S$. turgidiscabies, and that nec1 is involved in pathogenicity and physically linked to the thaxtomin A biosynthetic genes.

Additional keywords: horizontal gene transfer, pathogenicity determinants.

Streptomyces spp. are gram-positive, filamentous prokaryotes, many of which produce biologically active secondary metabolites. These organisms have DNA with a high proportion of guanine and cytosine and a single linear chromosome (Lin et al. 1993). Almost all Streptomyces spp. are soil-inhabiting saprophytes. However, a few species are pathogenic and cause diseases of underground structures of diverse plant species. Streptomyces scabies (Lambert and Loria 1989a), a cause of potato scab and similar diseases of tap

Corresponding author: Rosemary Loria; Telephone: 1-607-255-7831; Fax: 1-607-255-4471; E-mail: rl21@ cornell.edu

Nucleotide and/or amino acid sequence data have been deposited in the EMBL/GenBank data base under accession numbers AF073313, AF073314, AF073315, AF073316, AF073317, AF073318, AF073319, and AF073320. root crops, was the first described and is the best studied of the pathogenic species. Streptomyces acidiscabies (Lambert and Loria 1989b) produces symptoms like those of S. scabies (Manzer et al. 1977) and has a similar host range (Lambert and Loria 1990), although the two species are not closely related, based on DNA-DNA homology (Healy and Lambert 1991) and 16S rRNA sequences (Takeuchi et al. 1996). Another Streptomyces sp. that is pathogenic on potato has been described from the island of Hokkaido in Japan (Miyajima et al. 1998). This species, S. turgidiscabies, has a wide host range among root crops and produces symptoms like those of S. scabies on potato tubers, suggesting a common mechanism of pathogenicity. However, this recently described species is distinctly different from both $S$. acidiscabies and $S$. scabies, based on DNA-DNA homology and ribosomal DNA sequence comparisons, as well as morphological characteristics (Miyajima et al. 1998; Takeuchi et al. 1996).

Thaxtomins, a family of modified dipeptide phytotoxins, are produced by strains of $S$. scabies and $S$. acidiscabies and may play a role in the pathogenicity of these species on potato (King et al. 1991, 1989; Lawrence et al. 1990). Thaxtomins A and $\mathrm{B}$ were the first of the nine members of the family to be described. Thaxtomin A is the most abundant of these toxins in potato tuber tissue infected by S. scabies. When purified toxin is applied to plant tissue, it causes necrosis and cell hypertrophy, both of which are symptoms of tuber infection by S. scabies (Lawrence et al. 1990). Production of thaxtomins is perfectly correlated to pathogenicity in all strains tested to date including $S$. scabies, S. acidiscabies, and the sweet potato pathogen S. ipomoeae (King et al. 1994), suggesting that thaxtomins may be a common pathogenicity determinant among diverse Streptomyces spp. that cause plant disease.

Our goal is to identify plant pathogenicity determinants in Streptomyces. We cloned a gene, necl, from S. scabies 84.34 (ATCC 41973) that allows the nonpathogen Streptomyces lividans to necrotize and colonize potato tuber disks (PTDs) and produce scablike symptoms on immature potato tubers (Bukhalid and Loria 1997). S. lividans expressing necl does not produce thaxtomin A, but does produce an unidentified, extracellular, water-soluble compound that causes necrosis on potato tuber disks. No significant homologies to necl were found in the nucleic acid and protein data bases. necl has a low $\mathrm{G}+\mathrm{C}$ content $(54 \%)$, suggesting that it has moved horizontally from another genus. In S. scabies, necl is adjacent to an open reading frame (ORF), designated ORFtnp, that has a 
high level of identity to members of the Staphylococcus aureus IS256 family of transposases (Collins and Stephens 1991; Komeda et al. 1996; Rouch et al. 1987). This family of transposases is known to mobilize antibiotic resistance genes among clinically important bacteria.

Southern analysis of 10 strains of S. scabies and S. acidiscabies and 11 nonpathogenic Streptomyces spp. strains suggested that necl is associated with thaxtomin A production and linked to ORFtnp in Streptomyces strains that are pathogenic on potato (Bukhalid and Loria 1997). These data are consistent with the hypothesis that necl is involved in pathogenicity and was mobilized into $S$. scabies and $S$. acidiscabies, perhaps through a transposition event mediated by ORFtnp. The objectives of this research were (i) to test the hypothesis that the presence of necl is predictive of plant pathogenicity and thaxtomin A production in a large geographically diverse group of Streptomyces strains, including those of S. scabies, S. acidiscabies, and S. turgidiscabies; (ii) to determine if necl is consistently linked to ORFtnp in these strains, as this would suggest simultaneous mobilization of both genes; (iii) to determine if necl is functionally conserved in these strains by evaluating the phenotype of the necl homologs from plant-pathogenic strains of other Streptomyces spp., when expressed in the nonpathogen S. lividans; and, (iv) to evaluate DNA sequence divergence among necl and ORFtnp homologs from the three species, as a measure of the time over which horizontal gene transfer may have occurred.

\section{RESULTS}

\section{Evaluation of Streptomyces strains for thaxtomin A production and pathogenicity.}

Streptomyces strains were evaluated for production of the cyclic dipeptide phytotoxin, thaxtomin A, and their ability to produce disease on immature potato tubers. The morphological and physiological characteristics of phytopathogenic Streptomyces strains were consistent with species descriptions of either S. scabies, S. acidiscabies, or S. turgidiscabies; nonpathogenic species were not characterized. The pathogenicity of Streptomyces strains was assayed with a previously described potato tuber bioassay (Fig. 1). Thaxtomins were extracted from culture supernatants and fractionated by thin layer chromatography (TLC). The phytotoxicity of thaxtomin A-containing extracts was confirmed with a previously described PTD assay. All strains that were pathogenic also produced thaxtomin A (Table 1), as revealed by a yellow band comigrating with a thaxtomin A standard on TLC plates. These extracts were necrogenic on PTDs. None of the nonpathogenic strains produced thaxtomin A or were necrogenic on PTDs. All strains of $S$. turgidiscabies, a newly described phytopathogenic species from Japan (Miyajima et al. 1998), produced thaxtomin A (Table 1) and were pathogenic on potato tubers (Fig. 1).

\section{Presence of nec1 and ORFtnp homologs in Streptomyces strains.}

Genomic DNA from all strains was restricted with the endonuclease Bam HI and subjected to Southern analysis with internal cloned fragments of necl (305-bp PstI-RsaI fragment) and ORFtnp (490-bp SstII-ClaI fragment) as probes (Bukhalid and Loria 1997). DNA from all 43 phytopathogenic strains hybridized to a single fragment when necl was used as a probe (Fig. 2), except for a single $S$. scabies strain from South Africa, CEK-018, that is pathogenic and produces thaxtomin A (Fig. 1). None of the nonpathogenic strains had necl homologs. The size of the fragment hybridizing to necl varied among the 23 S. scabies strains. Strains of S. acidiscabies and $S$. turgidiscabies did not vary significantly in their hybridization patterns.

The ORFtnp probe strongly hybridized to one restriction fragment in all of the S. scabies, S. acidiscabies, and $S$. turgidiscabies strains tested. In all the $S$. scabies strains, however, ORFtnp weakly hybridized to one additional restriction fragment. Unlike necl, ORFtnp homologs were present in some of the nonpathogens; Streptomyces spp. strains DHL-1, 88.24 , and TO-2, which were originally isolated from scab lesions on potato, had a single copy of ORFtnp (Table 1; Fig. 2). S. scabies strains were also diverse in their hybridization patterns to ORFtnp, while $S$. acidiscabies and S. turgidiscabies strains did not vary.

Southern analysis was consistent with linkage of necl and ORFtnp in the phytopathogenic strains tested. The BamHI digest demonstrated that ORFtnp and necl are on the same restriction fragment in the $S$. scabies and the $S$. turgidiscabies strains (Fig. 2). However, there is a BamHI restriction site in the necl/ORFtnp intergenic region in S. acidiscabies. Southern blot analysis with the restriction endonuclease $P v u \mathrm{II}$, which does not have a site in the intergenic region, confirmed the linkage of ORFtnp and necl in S. acidiscabies (data not shown).

\section{The sequence of nec1 in $S$. scabies, $S$. acidiscabies,} and $S$. turgidiscabies strains is identical.

To evaluate sequence divergence among necl homologs present in strains of the three pathogenic species, oligonucleotide primers specific to the sequences of necl and ORFtnp

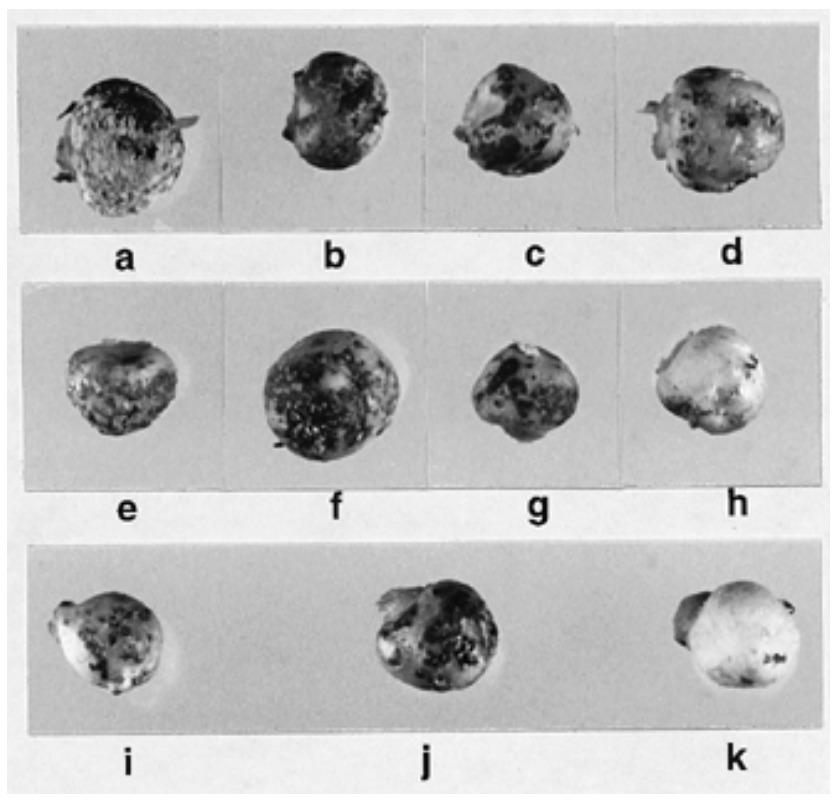

Fig. 1. Immature potato tubers infected with (a-c) Streptomyces turgidiscabies (Hi-C-20, Ko-g-6, Ko-g-2) and (d) S. scabies (SB-742) strains from Japan, (e-h) S. scabies (CEK-038A, CEK-038B, CEK037B, CEK-018) from South Africa, and (i, j) S. scabies (84.34, 87.22) from the U.S. Nonpathogen S. lividans TK24 (k) included as a control. 
were designed. The coding sequences of these two genes were polymerase chain reaction (PCR)-amplified from two $S$. scabies strains, ATCC 33282 and 87.22, two S. acidiscabies strains, ATCC 49003 and 84.104, and two S. turgidiscabies strains, Hi-C-13 and Car-8. The sequence of the primers for $n e c l$, $\mathrm{Nf}$ and $\mathrm{Nr}$, and for ORFtnp, Tf and $\mathrm{Tr}$, was based on the sequence from S. scabies strain 84.34 (ATCC 41973) (Bukhalid and Loria 1997) (Fig. 3). The predicted 0.72-kb and $0.55-\mathrm{kb}$ products for the coding sequences of necl and ORFtnp, respectively, were obtained from all six strains. A second set of primers, If and Ir, was used to amplify the intergenic region; the predicted $0.57-\mathrm{kb}$ products were obtained

Table 1. Streptomyces strains and their country of origin, hosts, pathogenicity on immature potato tubers, thaxtomin A production, and hybridization to ORFtnp and necl

\begin{tabular}{|c|c|c|c|c|c|c|}
\hline \multirow[b]{2}{*}{ Strain $^{a}$} & \multirow[b]{2}{*}{ Origin $^{b}$} & \multirow[b]{2}{*}{ Host } & \multirow[b]{2}{*}{ Pathogenicity on PMT, } & \multirow[b]{2}{*}{ Thaxtomin A production ${ }^{d}$} & \multicolumn{2}{|c|}{ Hybridization } \\
\hline & & & & & necl & ORFtmp \\
\hline \multicolumn{7}{|l|}{ S. scabies } \\
\hline 84-34 (ATCC 41973)T & United States & Potato & + & + & + & + \\
\hline $84-102$ & United States & Potato & + & + & + & + \\
\hline $87-22$ & United States & Potato & + & + & + & + \\
\hline 87 & United States & Potato & + & + & + & + \\
\hline 89 & United States & Potato & + & + & + & + \\
\hline Pon-P & United States & Potato & + & + & + & + \\
\hline ATCC 33282 & Hungary & Potato & + & + & + & + \\
\hline SB-742 & Japan & Potato & + & + & + & + \\
\hline S-7 & Japan & Potato & + & + & + & + \\
\hline CEK-010 & South Africa & Potato & + & + & + & + \\
\hline CEK-018 & South Africa & Potato & + & + & - & - \\
\hline CEK-037A & South Africa & Potato & + & + & + & + \\
\hline CEK-037B & South Africa & Potato & + & + & + & + \\
\hline CEK-038A & South Africa & Potato & + & + & + & + \\
\hline CEK-038B & South Africa & Potato & + & + & + & + \\
\hline DNK-043 & South Africa & Potato & + & + & + & + \\
\hline DNK-034 & South Africa & Potato & + & + & + & + \\
\hline DNK-025A & South Africa & Potato & + & + & + & + \\
\hline DNK-005 & South Africa & Potato & + & + & + & + \\
\hline DNK-020 & South Africa & Potato & + & + & + & + \\
\hline DNK-G01 & South Africa & Peanuts & + & + & + & + \\
\hline DNK-007 & South Africa & Potato & + & + & + & + \\
\hline VNT-048 & South Africa & Potato & + & + & + & + \\
\hline \multicolumn{7}{|l|}{ S. acidiscabies } \\
\hline ATCC 49003T & United States & Potato & + & + & + & + \\
\hline $84-104$ & United States & Potato & + & + & + & + \\
\hline $85-06$ & United States & Potato & + & + & + & + \\
\hline $84-182$ & United States & Potato & + & + & + & + \\
\hline $84-45$ & United States & Potato & + & + & + & + \\
\hline \multicolumn{7}{|l|}{ S. turgidiscabies } \\
\hline S-2 & Japan & Potato & + & + & + & + \\
\hline SS-9 & Japan & Sugar beet & + & + & + & + \\
\hline SS-13 & Japan & Sugar beet & + & + & + & + \\
\hline SS-12 & Japan & Sugar beet & + & + & + & + \\
\hline Hi-C-13 & Japan & Carrot & + & + & + & + \\
\hline $\mathrm{Hi}-\mathrm{C}-16$ & Japan & Carrot & + & + & + & + \\
\hline Hi-C-19 & Japan & Carrot & + & + & + & + \\
\hline Hi-C-20 & Japan & Carrot & + & + & + & + \\
\hline Hi-C-36 & Japan & Carrot & + & + & + & + \\
\hline Ko-g-2 & Japan & Great burdock & + & + & + & + \\
\hline Ko-g-6 & Japan & Great burdock & + & + & + & + \\
\hline Car-3 & Japan & Carrot & + & + & + & + \\
\hline Car-8 & Japan & Carrot & + & + & + & + \\
\hline Hi-R-11 & Japan & Radish & + & + & + & + \\
\hline Hi-R-15 & Japan & Radish & + & + & + & + \\
\hline S. griseus ATCC10246 & NA & NA & - & - & - & - \\
\hline S. coelicolor M146 & NA & NA & - & - & - & - \\
\hline \multicolumn{7}{|l|}{ Streptomyces spp. } \\
\hline To-2 & Japan & Potato & - & - & - & + \\
\hline 88.24 & United States & Potato & - & - & - & - \\
\hline 88.25 & United States & Potato & - & - & - & - \\
\hline 83.40 & United States & Potato & - & - & - & - \\
\hline DHL-1 & United States & Potato & - & - & - & + \\
\hline DHL-2 & United States & Potato & - & - & - & - \\
\hline
\end{tabular}

${ }^{\mathrm{a}} \mathrm{T}$, Type strain of the species.

${ }^{\mathrm{b}} \mathrm{NA}$, not known or applicable.

${ }^{c}$ PMT, Potato minitubers.

$\mathrm{d}+$, thaxtomin A production, pathogenicity on potato tubers, and DNA hybridization; -, no activity or hybridization. 
from both $S$. scabies and $S$. turgidiscabies strains, while a 2.0$\mathrm{kb}$ product was obtained from the $S$. acidiscabies strains.

Alignment of sequences of the PCR-amplified necl and ORFtnp from the six strains with the sequence of their homologs from S. scabies 84.34 (Bukhalid and Loria 1997) demonstrated that both of these genes had identical sequences in all seven strains. The sequences of the intergenic region of the four S. scabies and S. turgidiscabies strains were also identical to that of strain 84.34. Interestingly, in the S. acidiscabies strains, the intergenic sequence was identical to that of strain 84.34, except that it also contained a $1.5-\mathrm{kb}$ IS element (Fig. 4) recently identified in pathogenic Streptomyces spp. (F. Healy, unpublished).

\section{The nec1 homologs from S. turgidiscabies} are functionally conserved.

DNA fragments encoding the necl homologs from $S$. turgidiscabies Hi-C-13 and Car-8 and S. scabies 87.22 were amplified with restriction site-modified primers. An oligonucleotide primer pair was designed so as to amplify the coding sequence in addition to $168 \mathrm{bp}$ upstream of the translational start codon of necl. BamHI and EcoRI sites were introduced $5^{\prime}$ of the forward (5'-TCCCGACGCCGGAGCAAACCC-3') and reverse (Nr) primers (Fig. 3), respectively. The PCR products were cloned in the Streptomyces/Escherichia coli shuttle plasmid vector, pUWL-KS (Wehmeier 1995), where the expression of necl is driven by its native promoter. $S$. lividans TK24 protoplasts were transformed with the necl constructs from the $S$. scabies and S. turgidiscabies strains. Transformants carrying plasmids encoding the necl homologs were necrogenic on PTDs (Fig. 5); nontransformed S. lividans and this strain transformed with the plasmid alone were not necrogenic.

\section{nec1-specific oligonucleotide primers amplify} the $0.7-\mathrm{kb}$ gene from all the nec 1 -containing strains.

The sequence identity of necl homologs in seven pathogenic strains of Streptomyces spp. suggested that the sequence

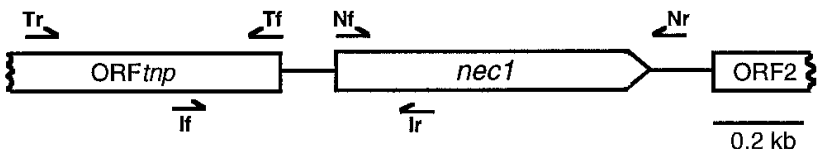

Fig. 3. Map of nec1, ORFtnp, and ORF2, and their respective oligonucleotide primers (half arrows).

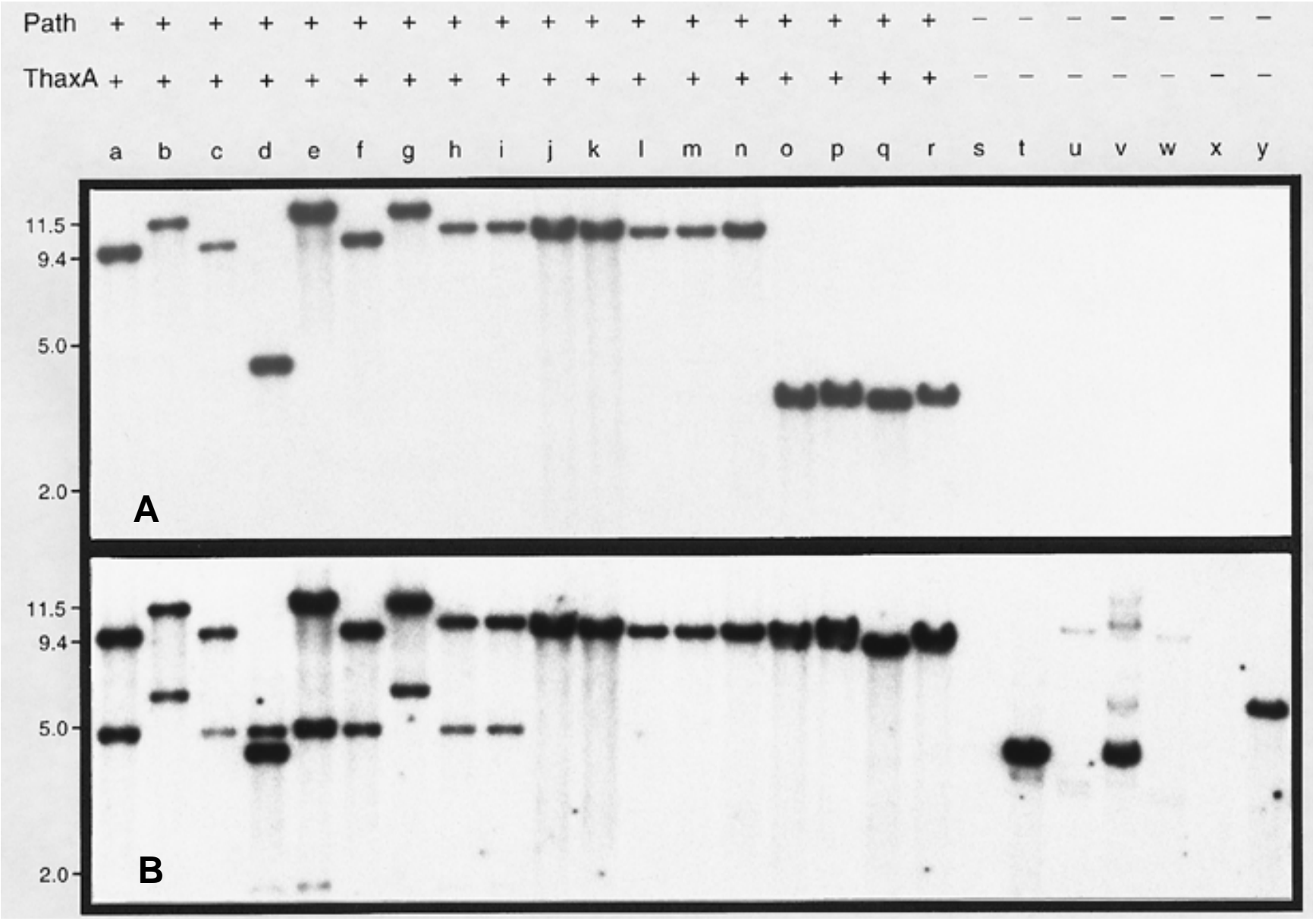

Fig. 2. Southern analysis of a BamHI digest of representative Streptomyces strains with internal fragments of (A) nec1 (305 bp) and (B) ORFtnp (490 bp). Plant-pathogenic strains included (a-i) S. scabies strains 84.34, 87.22, Pon-P, ATCC33282, S-7, SB-742, CEK-038A, DNK-007, VNT-048; (j-n) S. turgidiscabies strains S-2, SS-9, Hi-R-11, Ko-g-2, Hi-C-13, and (o-r) S. acidiscabies strains ATCC 49003, 84.104, 84.45, 84.182. Nonpathogenic strains included (s) S. coelicolor M146; (x) S. griseus ATCC10246, and (t-w, y) Streptomyces spp. strains DHL-1, DHL-2, 88.24, 88.25, To-2. Pathogenicity (Path) and thaxtomin A (ThaxA) production by strains are indicated. Size markers (kb) are shown to the left. 
of this gene is conserved in all strains that hybridize to necl. The oligonucleotide primers $\mathrm{Nf}$ and $\mathrm{Nr}$ were used to amplify the coding sequence of necl from the S. scabies, S. turgidiscabies, and $S$. acidiscabies strains listed in Table 1 . The predicted $0.7-\mathrm{kb}$ product was amplified from all of the pathogenic Streptomyces strains that hybridized to necl, demonstrating that these primers could be useful in the detection of pathogenic Streptomyces strains.

\section{DISCUSSION}

Our data support the hypothesis that thaxtomin A production is a pathogenicity determinant in plant-pathogenic Streptomyces spp. All strains that produced thaxtomin A were pathogenic on potato tubers and all strains that were pathogenic on potato tubers produced thaxtomin A. We included representative strains of three Streptomyces spp. known to be pathogenic on plants from four geographic locations. This is the most comprehensive analysis of the relationship between thaxtomin A production and pathogenicity yet reported, and the first report of thaxtomin A production by $S$. turgidiscabies. The data support the hypothesis that thaxtomins are hostnonselective pathogenicity determinants in plant-pathogenic Streptomyces spp. Phytotoxins produced by most plant prokaryotes are host-nonselective virulence, rather than pathogenicity, determinants (Gross 1991). In contrast, many fungal plant pathogens produce host-selective phytotoxins that are pathogenicity determinants (Walton 1996).

necl was structurally conserved among all but one of the pathogenic strains examined, based on Southern analysis and PCR amplification with necl-specific primers. Further, necl was functionally conserved among at least four strains representing S. scabies and S. turgidiscabies. This is not surprising, as our data demonstrated that the sequence of necl in these four strains and two additional strains of S. acidiscabies is identical. The genetic diversity of $S$. scabies, $S$. acidiscabies, and $S$. turgidiscabies is well established. Therefore, necl sequence identity among strains of these three species indicates that horizontal transfer of necl among these strains has occurred relatively recently. Further, the low $\mathrm{G}+\mathrm{C}$ content of necl indicates that this gene did not evolve in the genus Streptomyces, but was horizontally transferred into Streptomyces spp. from an organism with a $\mathrm{G}+\mathrm{C}$ content of approximately $55 \%$.

The function of necl is not presently known. The necrogenic phenotype of necl suggests that this gene plays a role in plant pathogenicity in streptomycetes. A previous study with fewer Streptomyces strains had found a perfect correlation between the presence of necl and thaxtomin A production
(Bukhalid and Loria 1997). In this study, we identified one thaxtomin A-producing strain, CEK-018, out of 43 that did not hybridize to necl. We believe that the absence of a necl homolog in CEK-018 indicates that necl is not directly involved in thaxtomin A biosynthesis but is, perhaps, a virulence factor in plant-pathogenic streptomycetes. However, the high correlation between the presence of necl and thaxtomin A production in this diverse group of Streptomyces strains does suggest physical linkage between necl and the thaxtomin A biosynthetic genes. Linkage would facilitate the simultaneous transfer of these genes on a mobile genetic element, such as a plasmid or a conjugative transposon.

The physical linkage of necl and ORFtnp in a large number of genetically and geographically diverse, plant-pathogenic Streptomyces strains suggests that ORFtnp may have been involved in the horizontal transfer of necl into Streptomyces

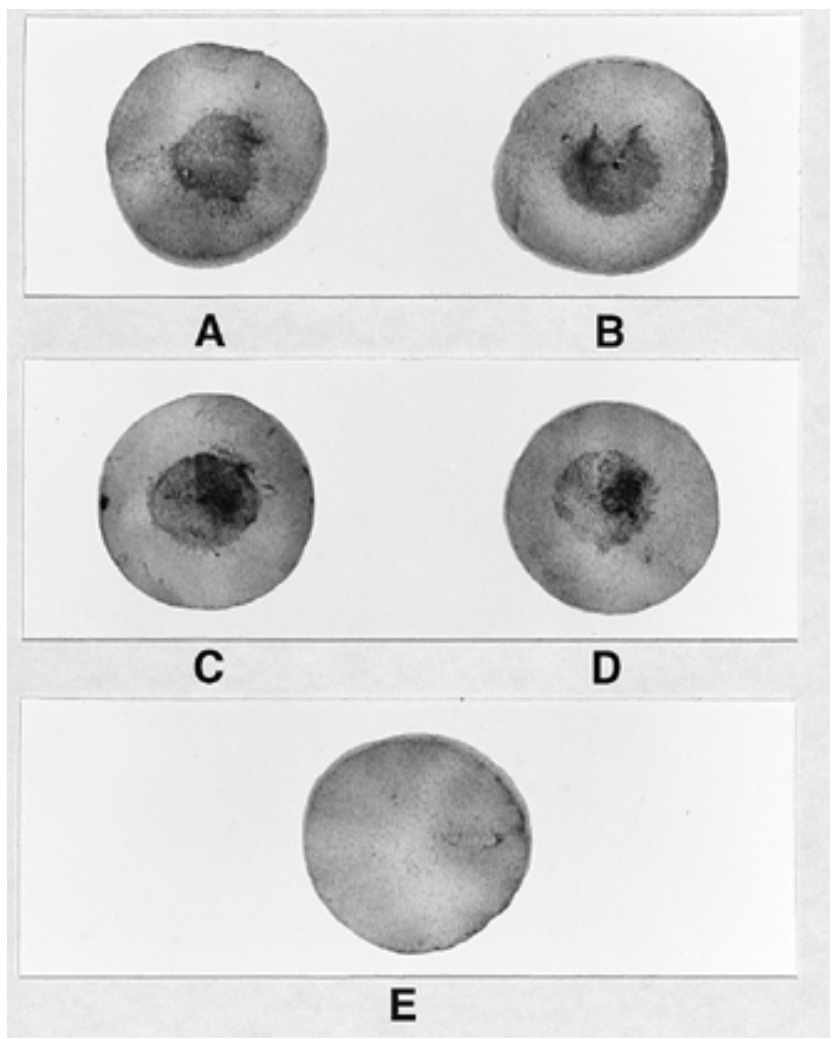

Fig. 5. Necrogenic phenotype on potato tuber disks (PTDs) of Streptomyces lividans TK24 expressing necl homologs from two strains of $S$. turgidiscabies, (A) Hi-C-13 and (B) Car-8, along with the disks inoculated with the wild-type strains (C) Hi-C-13 and (D) Car-8. E, S. lividans TK24 harboring the vector alone is included as a control.
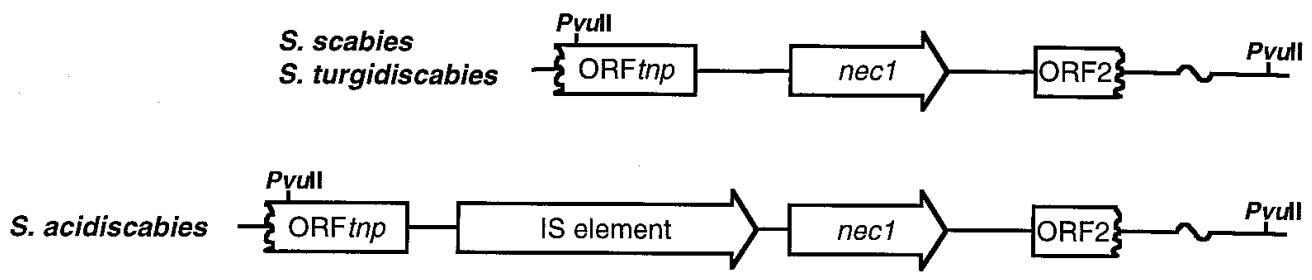

Fig. 4. Arrangement of nec1, ORFtnp, ORF2, and the IS element in Strepomyces scabies, S. acidiscabies, and S. turgidiscabies. Sequences of nec1 and ORFtnp are identical in strains representing all three species. Two PvuII restriction sites shown are conserved in at least four strains of each species, based on Southern analysis (data not presented). 
spp. ORFtnp is a transposase pseudogene with a high level of amino acid identity to the putative transposases of the IS1164 from Rhodococcus rhodochrous (68.2\%) and the IS1407 from Mycobacterium celatum (68.4\%), as well as other transposases of the Staphylococcus aureus IS256 family of transposons. Members of this family of transposons are implicated in horizontal movement of genes conferring a fitness advantage, such as antibiotic resistance genes, among clinically important bacteria (Rouch et al. 1987; Tauch et al. 1995), or are associated with genes involved in the biodegradation of toxic nitriles (Komeda et al. 1996). ORFtnp is an interrupted ORF; a frameshift mutation at nucleotide 245 introduced a stop codon in the $\mathrm{N}$ terminus of ORFtnp, reducing it to a 168 amino acid pseudogene. The frameshift-corrected sequence would encode a putative 413 amino acid polypeptide (data not presented) (Bukhalid and Loria 1997). The mutation in the coding sequence of ORFtnp might have played a major role in the stabilization of necl in the $S$. scabies genome. These data are consistent with the hypothesis that necl was mobilized into $S$. scabies, through a transposition event mediated by ORFtnp, and subsequently mobilized within a larger DNA fragment, containing ORFtnp, into other Streptomyces spp. through a different mechanism. Southern analysis also showed that a $P v u \mathrm{II}$ site in the sequence approximately $3.0 \mathrm{~kb}$ downstream of necl is also highly conserved (Fig. 4), indicating that a much larger DNA fragment may have been mobilized among pathogenic Streptomyces spp.

Hybridization patterns of necl and ORFtnp support other data indicating that $S$. scabies is a much more genetically diverse, and therefore more ancient, species than are $S$. acidiscabies and $S$. turgidiscabies. Our knowledge about the emergence of $S$. acidiscabies and $S$. turgidiscabies as economically important pathogens is also consistent with a relatively recent origin for these species. Strains that were eventually named $S$. acidiscabies were isolated in the 1940 s from potato scab lesions on potatoes grown in acidic soils (Manzer et al. 1977). Low soil $\mathrm{pH}$ had been used by potato growers to suppress S. scabies (Manzer et al. 1977) and may have facilitated transfer of genetic determinants for pathogenicity from $S$. scabies to the acid-tolerant species $S$. acidiscabies. S. turgidiscabies was recently described from the island of Hokkaido in Japan. S. scabies, which is also well established there, was likely introduced to Hokkaido with potato seed tubers from the northeastern United States, approximately 50 years ago. We expect that transfer of pathogenicity-associated genes from $S$. scabies to $S$. turgidiscabies occurred during the last 50 years on Hokkaido.

The apparent specificity of necl primers for three pathogenic Streptomyces spp. has practical applications. Plant disease diagnosticians now rely on potato tuber pathogenicity tests, a time-consuming and inconvenient assay, for determining the etiology of scablike diseases on tap root and tuber crops (Schaad 1988). If necl primers are specific for pathogenic strains of $S$. scabies, $S$. acidiscabies, and $S$. turgidiscabies, amplification of necl might substitute for pathogenicity testing. This tool, in combination with relatively simple morphological evaluations, could potentially be very useful in identifying species responsible for scab diseases. In addition, it may be possible to use necl primers for detection of these three species in soil, for studies on the ecology of plant-pathogenic Streptomyces spp., and for the development of disease prediction models based on pathogen population densities.

\section{MATERIALS AND METHODS}

Culture media and conditions.

Luria Bertani medium (LB; Sigma, St. Louis, MO) and the International Streptomyces Project Medium 2 (ISP2; Difco, Detroit, MI) were used for the routine cultivation of $E$. coli and Streptomyces strains, respectively. CRM medium (Pigac and Schrempf 1995) was used to culture Streptomyces strains for DNA extractions. Oatmeal broth (OMB) and oatmeal agar (OMA) were used to assess thaxtomin A production and to produce inoculum for pathogenicity tests (Loria et al. 1995). Spore suspensions of all the Streptomyces strains were prepared as previously described (Hopwood et al. 1985). Antibiotics were added to media at the following final concentrations: thiostrepton (Th), 25 to $50 \mathrm{mg} / \mathrm{liter}$; ampicillin (Ap), $100 \mathrm{mg} / \mathrm{liter}$.

\section{Streptomyces strains and their characterization.}

Strains of S. scabies, S. acidiscabies, S. turgidiscabies, and a number of nonpathogenic Streptomyces spp. were included in this study (Table 1). Strains were originally isolated from South Africa (14), Japan (18), Hungary (1), and the United States (16). Morphological and physiological characterization was based on methods of the International Streptomyces Project as described by Shirling and Gottlieb (1966). S. lividans TK24 was used for the expression of necl homologs from Streptomyces spp.

\section{Plant bioassays.}

Immature potato tubers (Loria and Kempter 1986) were used to evaluate pathogenic activity, as described in Loria et al. (1995), with the following modifications. Streptomyces strains were grown in OMB amended with trace elements (Hopwood et al. 1985). Mycelium was washed in sterile, distilled water by centrifugation to remove the preformed thaxtomins in the OMB media, and then resuspended before it was used as inoculum. Six immature potato tubers were immersed for $5 \mathrm{~min}$ in mycelial suspensions of the test strains, followed by incubation at $100 \%$ relative humidity for 3 to 4 days at 22 to $24^{\circ} \mathrm{C}$ in the dark. Potato tubers were scored for scab lesions. A previously described (Loria et al. 1995) PTD assay was used to screen $S$. lividans transformants and chloroform extracts of culture supernatants for necrotizing ability.

\section{Phytotoxin assay.}

Methods for evaluation of thaxtomin A production by Streptomyces strains in culture media were similar to those used previously (Loria et al. 1995). Thaxtomins were extracted from the supernatant of the OMB cultures used in the potato tuber bioassay and fractionated by TLC. Necrogenic activity of the chloroform extracts was also tested in the PTD assay. Bands of interest were removed from the TLC plates, eluted in methanol, concentrated, and tested for necrogenic activity on PTD.

\section{Recombinant DNA techniques.}

Recombinant DNA techniques were carried out according to the methods of Hopwood et al. (1985) and Rao et al. (1987) 
for Streptomyces and Sambrook et al. (1989) for E. coli, except for the preparation of competent $E$. coli DH5 $\alpha$ MCR cells (Hanahan 1985). For subcloning and preparation of DNA for probes, individual DNA restriction fragments were purified by separation on SeaKem GTG agarose gels (FMC BioProducts, Rockland, ME), followed by elution of DNA with GenElute columns (Supelco, Bellefonte, PA), according to the manufacturers' procedures. Restriction enzymes and DNA modification enzymes were purchased from GIBCO BRL (Grand Island, NY) and New England Biolabs (NEB; Beverly, MA).

\section{Southern blot analysis.}

Total genomic DNA was extracted by a miniprep modification of the procedure of Rao et al. (1987). DNA was digested with BamHI, separated by agarose gel electrophoresis, UVnicked, transferred to Hybond- $\mathrm{N}^{+}$nylon membranes (Amersham, Little Chalfont, England) by the manufacturer's alkaline transfer method, and hybridized to random-primed $\left[\alpha-{ }^{32} \mathrm{P}\right] \mathrm{dCTP}-$ labeled DNA probes made with the Prime-It II labeling kit from Stratagene (La Jolla, CA). Prehybridization and hybridization were done at $65^{\circ} \mathrm{C}$ overnight in the following buffer: $2 \times$ SSPE ( $1 \times$ SSPE is $0.18 \mathrm{M} \mathrm{NaCl}, 10 \mathrm{MM}$ $\mathrm{NaPO}_{4}$, and $1 \mathrm{M}$ EDTA [pH 7.7]), 5× Denhardt's, $1 \%$ bovine serum albumin (BSA), and 5\% sodium dodecyl sulfate (SDS; Amersham). The most stringent wash was $0.1 \times$ SSC $(1 \times$ SSC is $0.15 \mathrm{M} \mathrm{NaCl}$ plus $0.015 \mathrm{M}$ sodium citrate) and $0.5 \%$ SDS for $15 \mathrm{~min}$ at $65^{\circ} \mathrm{C}$. Autoradiography was performed with XOMAT AR X-ray film (Eastman Kodak) at $-80^{\circ} \mathrm{C}$.

\section{PCR amplification.}

Streptomyces cultures were grown in $5 \mathrm{ml}$ of CRM in test tubes for 24 to $48 \mathrm{~h}$ at $30^{\circ} \mathrm{C}$. DNA from $2 \mathrm{ml}$ of culture was extracted by washing mycelium in $1 \mathrm{ml}$ of sterile, distilled water, followed by boiling mycelium in $200 \mu \mathrm{l}$ of water, and DNA in the supernatant was recovered by centrifugation. The primers for necl and ORFtnp were $\mathrm{Nf}\left(5^{\prime}\right.$ ATGAGCGCGAACGGAAGCCCCGGA-3') and $\mathrm{Nr}$ (5'GCAGGTCGTCACGAA GGATCG-3 $\left.{ }^{\prime}\right)$ and Tf (5'ATGACCGCATCCGACA GTCTGCCC-3') and $\operatorname{Tr}\left(5^{\prime}-\right.$ TTGAT GATCCGGCCGCCCTCGC-3') (Fig. 3), respectively. The primers for the intergenic region were If $\left(5^{\prime}-\right.$ GTTGTCTTCGGCGAGGGCGTGCAGG-3') and Ir (5'AGCGGAAGGATT TGCGACCACAACG-3'). Amplification was carried out with Taq DNA polymerase (0.5 U; Perkin Elmer, Foster City, CA) or the high fidelity $p f u$ DNA polymerase (2.5 U; Stratagene) under the following conditions: DNA denaturing at $95^{\circ} \mathrm{C}$ for $1 \mathrm{~min}$, annealing at $60^{\circ} \mathrm{C}$ for 1 min, and elongation at $72^{\circ} \mathrm{C}$ for $2 \mathrm{~min}$ for 30 cycles. The amplified DNA was then run on $1 \%$ agarose gels. The PCR amplification experiments were performed twice with independent cultures for each strain.

\section{Heterologous expression of nec1.}

PCR-amplified DNA was digested with the BamHI/EcoRI restriction enzymes and purified by agarose gel-electrophoresis. The bands were cut out and the DNA recovered with GenElute spin columns (Supelco). DNA fragments were ligated to the Streptomyces/E. coli shuttle plasmid vector, pUWL-KS (Wehmeier 1995), and used to transform E. coli DH5 $\alpha$ MCR. DNA from each of four recombinant plasmids containing the expected inserts was then used to transform $S$. lividans TK24 protoplasts. Transformations were carried out in the presence of polyethylene glycol following the procedure of Rao et al. (1987). Four independent transformants were picked for each recombinant plasmid, cultured on oatmeal agar, and tested for necrogenic activity on PTD.

\section{Nucleotide sequencing and analysis.}

Cloned or pooled PCR products of necl homologs were sequenced by the dideoxy-chain termination method with an automated sequencer (DNA Services, Biotechnology, Cornell University, Ithaca, NY). The necl homologs from each strain were sequenced at least twice, from PCR products obtained from different DNA preparations, so as to eliminate the possibility of cross contamination of DNA templates. The T3 and T7 primers were used for sequencing both strands of the PCR products cloned in pUWL-KS, whereas the amplification reverse and forward primers were used for sequencing the pooled PCR products. PCR products were purified with the QIAquick columns from Qiagen (Chatsworth, CA). MEGALIGN and SEQMAN (DNASTAR, Madison, WI) were used for the multiple sequence alignments with the Clustal method and for sequence assembly and contig management, respectively.

\section{ACKNOWLEDGMENTS}

We would like to thank F. Kodama for providing us with the $S$. turgidiscabies and S. scabies strains from Japan; A. De Klerk for providing the $S$. scabies strains from South Africa; N. A. Anderson for providing the S. scabies strains Pon-P, 87, and 89; D. H. Lambert for providing strains DHL-1 and DHL-2; and K. Chater for providing $S$. coelicolor M146. This work was supported by USDA NRI grant 93-37303-89-83 and the New York State Agricultural Experiment Station, Hatch Project 153414 .

\section{LITERATURE CITED}

Bukhalid, R. B., and Loria, R. 1997. Cloning and expression of a gene from Streptomyces scabies encoding a putative pathogenicity factor. J. Bacteriol. 179:7776-7783.

Collins, D. M., and Stephens, D. M. 1991. Identification of an insertion sequence, IS1081, in Mycobacterium bovis. FEMS Microbiol. Lett. $83: 11-16$.

Gross, D. C. 1991. Molecular and genetic analysis of toxin production by pathovars of Pseudomonas syringae. Annu. Rev. Phytopathol. 29: 247-278.

Hanahan, D. 1985. Techniques for transformation of Escherichia coli. Pages 109-135 in : DNA Cloning: A Practical Approach. Vol. 1. D. M. Glover, ed. ILR Press, Washington, DC.

Healy, F. G., and Lambert, D. H. 1991. Relationships among Streptomyces spp. causing potato scab. Int. J. Syst. Bacteriol. 41:479-482.

Hopwood, D. A., Bibb, M. J., Chater, K. F., Kieser, T., Burton, C. J., Kieser, H. M., Lydiate, D. J., Smith, C. P., Ward, J. M., and Schrempf, H. 1985. Genetic Manipulation of Streptomyces: A Laboratory Manual. The John Innes Foundation, Norwich, England.

King, R. R., Lawrence, C. H., Calhoun, L. A., and Ristaino, J. B. 1994. Isolation and characterization of thaxtomin-type phytotoxins associated with Streptomyces ipomoeae. J. Agric. Food Chem. 42:1791-1794.

King, R. R., Lawrence, C. H., and Clark, M. C. 1991. Correlation of phytotoxin production with pathogenicity of Streptomyces scabies isolates from scab infected potato tubers. Am. Potato J. 68:675-680.

King, R. R., Lawrence, C. H., Clark, M. C., and Calhoun, L. A. 1989. Isolation and characterization of phytotoxins associated with Streptomyces scabies. J. Chem. Soc. Chem. Commun. 13:849-850.

Komeda, H., Kobayashi, M., and Shimizu, S. 1996. Characterization of the gene cluster of high-molecular-mass nitrile hydratase (H-NHase) induced by its reaction product in Rhodococcus rhodochrous J1. Proc. 
Natl. Acad. Sci. USA 93:4267-4272.

Lambert, D. H., and Loria, R. 1989a. Streptomyces scabies sp. nov., nom. rev. Int. J. Syst. Bacteriol. 39:387-392.

Lambert, D. H., and Loria, R. 1989b. Streptomyces acidiscabies sp. nov. Int. J. Syst. Bacteriol. 39:393-396.

Lambert, D. H., and Loria, R. 1990. Taxonomy of streptomycetes causing potato scab. (Abstr.) Phytopathology 80:120-121.

Lawrence, C. H., Clark, M. C., and King, R. R. 1990. Induction of common scab symptoms in aseptically cultured potato tubers by the vivotoxin, thaxtomin. Phytopathology 80:606-608.

Lin, Y.-S., Kieser, H. M., Hopwood, D. A., and Chen, C. W. 1993. The chromosomal DNA of Streptomyces lividans 66 is linear. Mol. Microbiol. 10:923-933.

Loria, R., Bukhalid, R. A., Creath, R. A., Leiner, R. H., Olivier, M., and Steffens, J. C. 1995. Differential production of thaxtomins by pathogenic Streptomyces species in vitro. Phytopathology 85:537-541.

Loria, R., and Kempter, B. A. 1986. Relative resistance of potato tubers produced from stem cuttings and seed-piece-propagated plants to Streptomyces scabies. Plant Dis. 70:1146-1148.

Manzer, F. E., McIntyre, G. A., and Merriam, D. C. 1977. A new potato scab problem in Maine. Univ. Maine Orono Life Sci. Agric. Exp. Stn. Tech. Bull. 85:3-24.

Miyajima, K., Tanaka, F., Takeuchi, T., and Kuninaga, S. 1998. Streptomyces turgidiscabies sp. nov. Int. J. Syst. Bacteriol. 48:495-502.

Pigac, J., and Schrempf, H. 1995. A simple and rapid method of transformation of Streptomyces rimosus R6 and other streptomycetes by electroporation. Appl. Environ. Microbiol. 61:352-356.
Rao, R. N., Richardson, M. A., and Kuhstoss, S. 1987. Cosmid shuttle vectors for cloning and analysis of Streptomyces DNA. Methods Enzymol. 153:166-198.

Rouch, D. A., Byrne, M. E., Kong, Y. C., and Skurray, R. A. 1987. The aacA-aphD gentamicin and kanamycin resistance determinant of Tn4001 from Staphylococcus aureus: Expression and nucleotide sequence analysis. J. Gen. Microbiol. 133:3039-3052.

Sambrook, J., Fritsch, E. F., and Maniatis, T. A. 1989. Molecular Cloning: A Laboratory Manual. 2nd ed. Cold Spring Harbor Laboratory, Cold Spring Harbor, NY.

Schaad, N. W., ed. 1988. Laboratory Guide for Identification of Plant Pathogenic Bacteria. 2nd ed. American Phytopathological Society, St. Paul, MN.

Shirling, E. B., and Gottlieb, D. 1966. Methods for characterization of Streptomyces species. Int. J. Syst. Bacteriol. 16:313-340.

Takeuchi, T., Sawada, H., Tanaka, F., and Matsuda, I. 1996. Phylogenetic analysis of Streptomyces spp. causing potato scab based on $16 \mathrm{~S}$ rRNA sequences. Int. J. Syst. Bacteriol. 46:476-479.

Tauch, A., Kassing, F., Kalinowski, J., and Puehler, A. 1995. The Corynebacterium xerosis composite transposon Tn5432 consists of two identical insertion sequences, designated IS1249, flanking the erythromycin resistance gene ermCX. Plasmid 34:119-131.

Walton, J. D. 1996. Host-selective toxins: Agents of compatibility. Plant Cell 8:1723-1733.

Wehmeier, U. F. 1995. New multifunctional Escherichia coliStreptomyces shuttle vectors allowing blue-white screening on XGal plates. Gene 165:149-150. 